\title{
Telangiectasia Hemorrágica Hereditária: Proposta de Protocolo de Referenciação Interdisciplinar
}

\author{
Hereditary Hemorrhagic Telangiectasia: Proposal of a \\ Multidisciplinary Referral Guideline
}

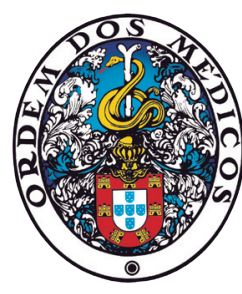

Isabel COSTA ${ }^{1}$, Inês GONÇALVES², Sofia MENDES ${ }^{3}$, Maria João ARAÚJO ${ }^{4}$, Regina CALDAS 5 , Berta RODRIGUES¹, Luís DIAS ${ }^{1}$

Acta Med Port 2021 Dec;34(12):876-881 - https://doi.org/10.20344/amp.14934

Palavras-chave: Telangiectasia Hemorrágica Hereditária/diagnóstico; Telangiectasia Hemorrágica Hereditária/tratamento

Keywords: Telangiectasia, Hereditary Hemorrhagic/diagnosis; Telangiectasia, Hereditary Hemorrhagic/therapy

\section{INTRODUÇÃO}

A telangiectasia hemorrágica hereditária $(\mathrm{THH})$, é uma doença da angiogénese, caracterizada pelo aparecimento de estruturas vasculares anómalas, designadas por malformações vasculares (MAV). Trata-se de uma doença rara, de transmissão autossómica dominante e multissistémica, cuja clínica se relaciona com os locais tipicamente mais afectados (pele, mucosas e órgãos viscerais). A epistáxis recorrente é a manifestação mais comum, ocorre em $95 \%$ dos casos e constitui o factor mais limitante na qualidade de vida dos doentes. ${ }^{1}$ Apesar de apresentarem uma esperança média de vida igual à da população em geral, sabe-se que doentes com THH apresentam pior qualidade de vida. Zarrabeitia et al (2017) mostrou que a mesma está afectada em todas as dimensões avaliadas no seu estudo (mobilidade, auto-cuidado, actividades da vida diária, dor/desconforto e ansiedade/depressão), apresentando pontuações semelhantes a outras doenças crónicas. Os doentes mais velhos, do sexo masculino e portadores de mutações ACVRL1 parecem apresentar pior qualidade de vida. $^{2}$

Os doentes com suspeita de THH são frequentemente referenciados pelos cuidados de saúde primários, para vigilância em consulta hospitalar. Como doença multiorgânica, a sua abordagem deve ser multidisciplinar. Programas de rastreio de indivíduos assintomáticos e algumas recomendações terapêuticas têm sido propostos por alguns autores. No entanto, a dúvida, nem sempre fácil de esclarecer, centra-se desde sempre na seguinte questão: "quando e a quem devemos referenciar os doentes com esta patologia?".

Como tal, os autores passam a apresentar uma proposta de protocolo hospitalar de actuação clínica baseada na sua experiência clínica com 56 indivíduos com o diagnóstico confirmado de THH seguidos no Hospital de Braga aliada a uma revisão extensa das últimas recomendações disponíveis na literatura acerca do diagnóstico, abordagem e tratamento desta patologia. Neste projecto foram incluídas recomendações de várias especialidades hospitalares (Medicina Interna, Otorrinolaringologia, Gastroenterologia, Pneumologia e Dermatologia). As características da população seguida e tratada na nossa instituição, assim como a nossa experiência clínica até à data de publicação do presente artigo encontram-se resumidamente listadas na Tabela 1.

\section{Proposta de protocolo}

\section{Diagnóstico clínico e molecular}

A THH pode ser diagnosticada através da história clínica e do exame objectivo, utilizando os critérios de Curaçao. ${ }^{3} \mathrm{O}$ diagnóstico pode também ser confirmado através da identificação de mutações genéticas em ENG, ACVRL1 ou no gene SMAD4 (mothers against decapentaplegic homolog 4). ${ }^{1,4}$ No entanto, o estudo genético não é necessário para realizar o diagnóstico.

\section{Estudo e seguimento}

Pela natureza multissistémica da THH, os autores recomendam o seguimento em consulta de Medicina Interna. A avaliação ideal destes doentes inclui a pesquisa regular de sinais e sintomas associados às principais repercussões sistémicas da doença, tais como:

2.1. Défice de ferro/anemia: está indicada a monitorização periódica da hemoglobina $(\mathrm{Hb})$, hematócrito, reticulócitos e ferritina, assim como a avaliação da necessidade de suplementação de ferro ou transfusão de sangue.

2.2. Epistáxis: a escala Epistaxis Severity Score (Tabela $2)^{5}$ deve ser aplicada a todos os doentes, tendo em vista a correta estratificação da doença. ${ }^{6}$

2.3. Hemorragia gastrointestinal: A frequência de hemorragia do foro gastrointestinal $(\mathrm{Gl})$ na THH é de

\footnotetext{
1. Serviço de Otorrinolaringologia e Cirurgia de Cabeça e Pescoço. Hospital de Braga. Braga. Portugal.

2. Serviço de Medicina Interna. Hospital de Braga. Braga. Portugal.

3. Serviço de Gastroenterologia. Hospital de Braga. Braga. Portugal.

4. Serviço de Pneumologia. Hospital de Braga. Braga. Portugal.

5. Serviço de Dermatologia e Venereologia. Hospital de Braga. Braga. Portugal.

$\triangle$ Autor correspondente: Isabel Costa. isabelcostaorl@gmail.com

Recebido: 16 de novembro de 2020 - Aceite: 09 de fevereiro de 2021 - First published: 28 de abril de 2021 - Online issue published: 02 de dezembro de 2021 Copyright $\odot$ Ordem dos Médicos 2021
} 
Tabela 1 - Características sociodemográficas e clínicas da população com diagnóstico confirmado de THH seguida na instituição

\begin{tabular}{|c|c|}
\hline Idade actual (anos) & $62,3(40-86)$ \\
\hline Idade de diagnóstico (anos) & $33,9(12-53)$ \\
\hline Critérios de Curaçau presentes & $\%$ \\
\hline 4 & 52 \\
\hline 3 & 48 \\
\hline 2 & 0 \\
\hline 1 & 0 \\
\hline Epistaxis & 100 \\
\hline Lesões viscerais & 72 \\
\hline Telangiectasias mucocutâneas & 80 \\
\hline História familiar & 88 \\
\hline \multicolumn{2}{|l|}{ Familiares de $1^{\circ}$ grau afectados } \\
\hline 0 & 12 \\
\hline $1-2$ & 20 \\
\hline $3-5$ & 28 \\
\hline$>5$ & 40 \\
\hline \multicolumn{2}{|l|}{ Atingimento orgânico (para além do foro ORL) } \\
\hline Nenhum & 8 \\
\hline Gastrointestinal & 52 \\
\hline Pulmonar & 12 \\
\hline Cerebral & 20 \\
\hline Hepático & 4 \\
\hline Dermatológico & 24 \\
\hline \multicolumn{2}{|l|}{ Tratamento da epistáxis disponíveis/realizados } \\
\hline Sem necessidade de tratamento/medidas conservadoras & $35,7(n=20)$ \\
\hline Coagulação a laser & $5,4(n=3)$ \\
\hline Embolização nasal & $35,7(n=20)$ \\
\hline Radioterapia hemostática & $21,4(n=12)$ \\
\hline Anticorpo monoclonal anti-VEGF & $1,8(n=1)$ \\
\hline
\end{tabular}

aproximadamente $25 \%$. Os autores aconselham avaliação endoscópica do trato GI no caso de anemia desproporcional à severidade da epistáxis, hemorragia evidente do foro GI, ou no caso de dúvidas no diagnóstico. , $^{7}$

2.4. MAV hepáticas: na presença de sinais e sintomas sugestivos, o estudo deve ser iniciado com ecografia abdominal com estudo Doppler ou TC abdominal. Nos doentes assintomáticos, a pesquisa de MAV hepáticas é controversa, podendo ser realizada para suportar o diagnóstico de $\mathrm{THH} .{ }^{4,7}$

2.5. MAV pulmonares: as MAV pulmonares ocorrem em $30 \%-50 \%$ dos indivíduos com THH. Está recomendada a pesquisa de MAV pulmonares em todos os casos confirmados com $\mathrm{THH}$. O rastreio em doentes assintomáticos deve ser realizado através de ecocardiograma transtorácico contrastado com solução salina agitada. Na presença de sintomas, deverá ser pedido o estudo com tomografia computorizada (TC) do tórax com contraste. Nestes casos, perante a confirmação de um shunt pulmonar direito-esquerdo, os doentes devem realizar posteriormente estudo com TC tórax ou angio-TC. Em caso de estudo negativo, está recomendado novo rastreio dentro de cinco anos (ou mais precocemente se início de sintomas/eventos pulmonares). Doentes com o diagnóstico confirmado de MAV pulmonares devem receber profilaxia antibiótica previamente a procedimentos dentários, outros procedimentos não estéreis ou cirurgias. ${ }^{4,7,8}$

2.6. MAV cerebrais: a nivel cerebral, as MAV estão presentes desde o nascimento e ocorrem em cerca de $10 \%$ - $12 \%$ dos casos. Nos doentes assintomáticos, a pesquisa de MAV cerebrais deve ser ponderada se história familiar de hemorragia cerebral, mutação genética em ENG e SMAD4 e após discussão dos riscos e benefícios com o doente (Fig. 1). Por outro lado, alguns autores defendem que o rastreio de MAV cerebrais deve ser realizado o mais precocemente possível no caso de diagnóstico confirmado, preferencialmente no primeiro ano de vida. A sua confirmação diagnóstica deverá ser feita com recurso à ressonância magnética (RM) cerebral com contraste de gadolíneo. ${ }^{3-6,8} \mathrm{~A}$ avaliação por Neurorradiologia e Neurocirurgia dita a necessidade de tratamento..$^{9,10}$ 
Tabela 2 - Epistaxis severity score (adaptado de Hoag JB et al, 2010) ${ }^{12}$

a. Com que frequência apresenta epistaxis?

0. $<$ mensalmente

1. 1 vez por mês

2. 1 vez por semana

3. Várias vezes por semana

4. 1 vez por dia

5. Várias vezes por dia

b. Quanto tempo costuma durar um episódio de epistaxis?

0. $<1$ minuto

1. 1 - 5 minutos

2. 6 - 15 minutos

3. 16 - 30minutos

6. $>30$ minutos

c. Como descreveria a intensidade típica dos episódios?

0. Não é tipicamente profusa

1. Tipicamente profusa

d. Alguma vez recorreu a ajuda médica por epistaxis?

0. Não

1. Sim

e. Está neste momento anémico?

0. Não

1. Sim

f. Alguma vez recebeu uma transfusão (especificamente por epistaxis)?

0. Não

1. Sim

\subsection{Considerações especiais}

- Grávidas: a gravidez em mulheres com THH deverá ser considerada de alto risco. Durante a gravidez assiste-se geralmente a um crescimento das MAV pulmonares. Como tal, e se possível, as mesmas deverão ser pesquisadas e tratadas antes do início da mesma. Em mulheres a quem não foi excluída a presença de MAV cerebrais, deverá ser evitado o prolongamento da segunda fase do parto., 4

- Crianças: é importante que os pais ou cuidadores conheçam os sinais de alarme, para que os sintomas sejam rapidamente investigados e controlados. Em crianças assintomáticas, a decisão de investigar MAV é controversa, uma vez que se desconhece o risco de complicações ou o seu impacto na história natural da doença. Alguns autores advogam o rastreio de MAV cerebrais logo na infância através de RM cerebral, com repetição do estudo após o início da puberdade. ${ }^{4,7,9}$

2.8. Avaliação da qualidade de vida

Deverá ser realizada uma avaliação regular do impacto da THH na qualidade de vida dos doentes seguidos em consulta hospitalar. A repercussão a nível físico, psicológico e emocional dos sintomas, assim como as consequências a nível profissional e social na vida dos doentes deverá ser avaliado através de questionários devidamente validados
Pesquisar sinais e sintomas sugestivos em todos os casos

Se:

- sintomas presentes;

- história familiar de hemorragia cerebral;

- mutações genéticas ENG and SMAD4.

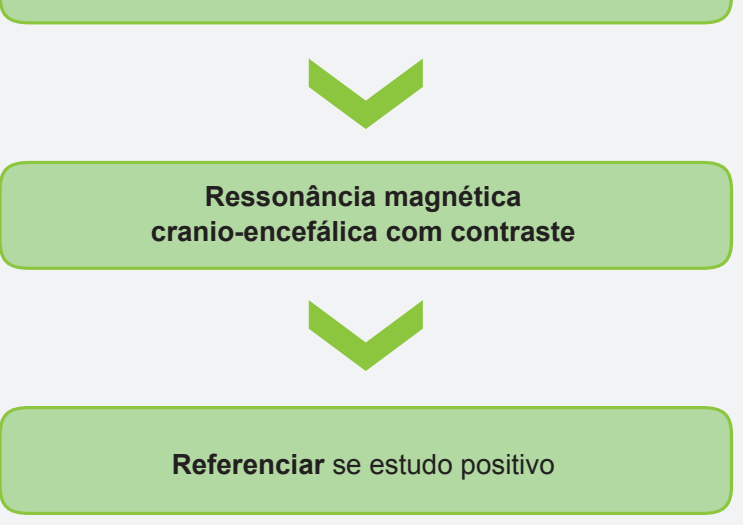

Figura 1 - Fluxograma de investigação de MAV cerebrais e de referenciação a Neurocirurgia 
(ex. EuroQol 5D-3L).

\section{Referenciação inter-especialidade}

3.1. Otorrinolaringologia: todos os doentes deverão ser orientados para consulta de ORL. O modus operandi para os doentes com THH inclui a avaliação sistematizada dos episódios de epistáxis por parte do otorrinolaringologista. $\mathrm{O}$ escalonamento terapêutico deve ser realizado consoante os diferentes graus de gravidade da doença (Tabela 1). A prevenção dos episódios de epistáxis constitui o pilar do controlo da doença e aplica-se a todos os graus de gravidade. A humidificação e hidratação da mucosa nasal, a evicção de fatores desencadeantes e o aconselhamento dietético devem fazer parte da abordagem primária ao doente com $\mathrm{THH}$. Na doença ligeira o tratamento deve ser essencialmente tópico, através de pomadas hemostáticas e antibióticas. ${ }^{10} \mathrm{Na}$ fase de doença moderada, a coagulação com laser é uma das opções recomendadas em vários centros de referência, sendo benéfico tanto nas lesões da mucosa nasal como da cavidade oral. Também o bevacizumab tópico (em pomada ou injectável) é uma opção segura, apesar de apresentar eficácia ainda controversa. ${ }^{11}$ Para a doença severa, os recursos terapêuticos são limitados e com resultados pouco consistentes. O bevacizumab intravenoso tem assumido um papel crescente no controlo da doença moderada a grave, pelo que os autores recomendam o seu uso em casos refractários e muito seleccionados. A oclusão nasal é uma opção cirúrgica que deve ser considerada na doença moderada a grave, crónica e refractária a outras medidas. ${ }^{1,4,7}$
3.2. Gastroenterologia: para o diagnóstico de MAV hepáticas, está indicada a realização de ecografia abdominal com estudo Doppler ou TC abdominal. ${ }^{7}$ Em caso de confirmação diagnóstica ou nos casos de necessidade de tratamento endoscópico de telangiectasias GI, o doente deverá ser referenciado para consulta de Gastrenterologia (Fig. 2). Nos doentes com o diagnóstico de THH e mutação no gene SMAD4 está recomendada a referenciação a consulta de Gastroenterologia para o rastreio de polipose juvenil e neoplasia GI. Nestes casos, deverá ser realizada colonoscopia e endoscopia digestiva alta a cada 1 - 2 anos a partir dos 12 - 15 anos de idade. ${ }^{4,7}$

3.3. Pneumologia: A avaliação por Pneumologia está indicada no caso de aparecimento de complicações associadas às MAV pulmonares, nomeadamente hemoptises e hemotórax (Fig. 3). Não obstante, na presença de sintomas e sinais sugestivos de hemoptises, qualquer médico deverá reencaminhar o doente para realização urgente de angio-TC torácico. Isto aplica-se também a situações de emergência, uma vez que este exame permite identificar o local da hemorragia, possíveis causas de hemorragia e permite um melhor planeamento do tratamento.

3.4. Dermatologia: O reconhecimento do padrão típico das telangiectasias cutâneas na THH é essencial para um diagnóstico mais precoce da doença (Fig. 4). ${ }^{12}$ Nos doentes com diagnóstico estabelecido de $\mathrm{THH}$, a referenciação para tratamento das lesões cutâneas está indicada nos casos sintomáticos (hemorragia e/ou dor) ou se o impacto estético interferir negativamente com a qualidade de vida do doente.

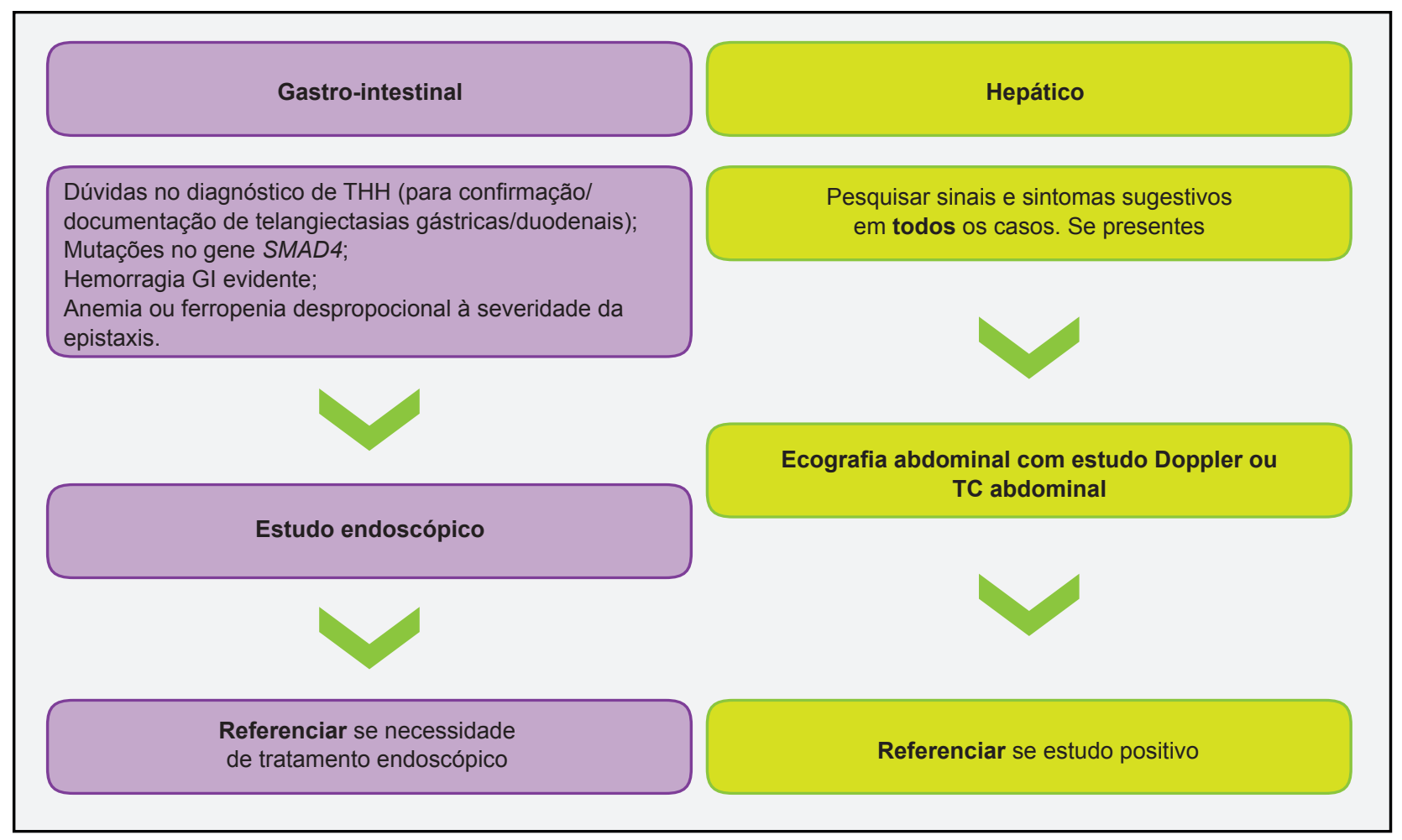

Figura 2 - Fluxograma de investigação e referenciação à consulta de Gastroenterologia 


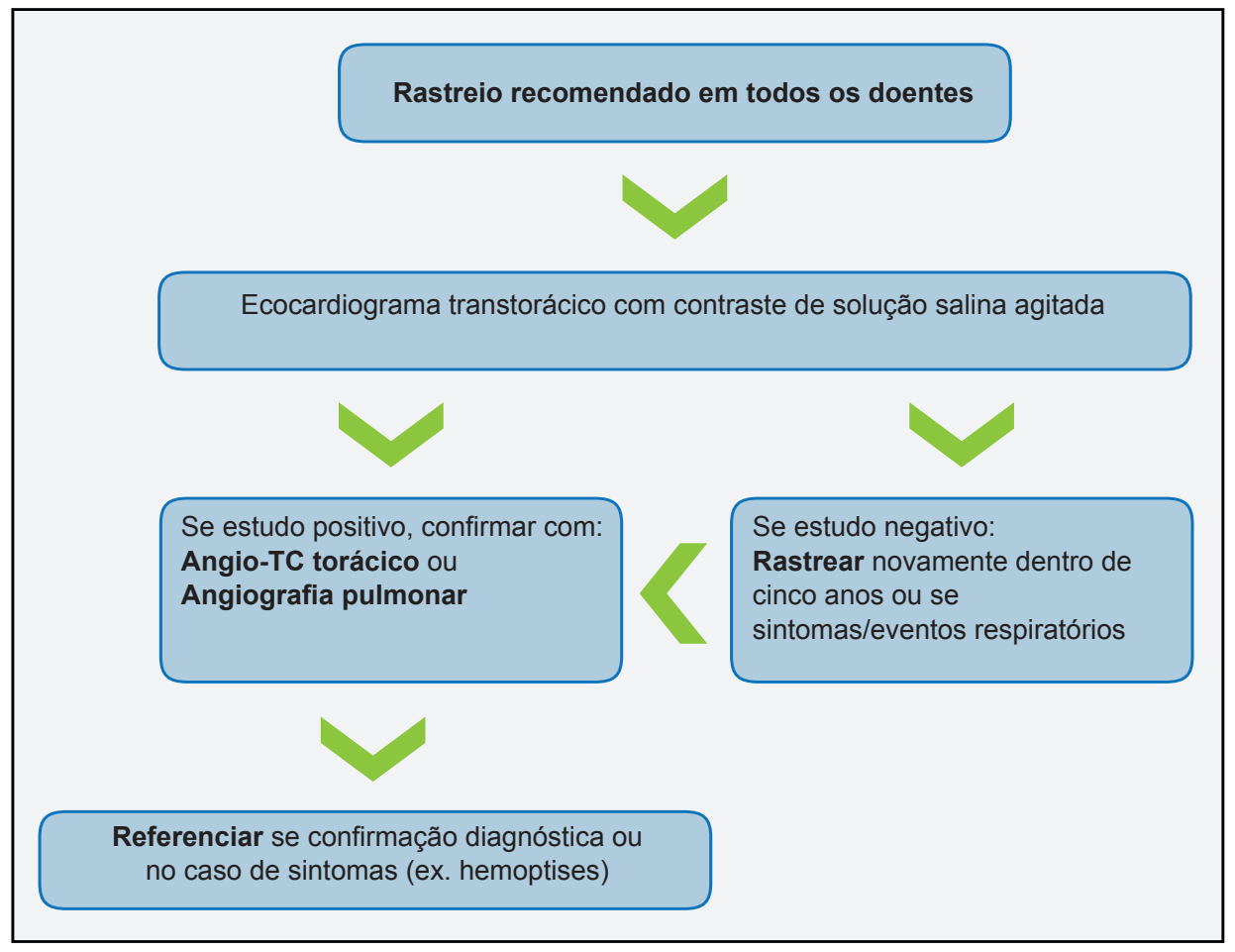

Figura 3 - Fluxograma de investigação e referenciação à consulta de Pneumologia

3.5. Genética médica: A THH é uma doença autossómica dominante, com penetrância e fenótipo variável. Apesar de não ser necessário para o diagnóstico, o estudo genético deve ser ponderado em todos os doentes com suspeita ou diagnóstico desta patologia (Fig. 5). O diagnóstico genético em doentes assintomáticos ou paucissintomáticos, mas com familiares de primeiro grau com mutação para THH conhecida não é consensual, pelo que deve ser discutido com o doente e avaliado individualmente. Sugere-se, no entanto, a referenciação de possíveis portadores para rastreio pré-concepcional e de crianças com progenitores afetados, para diagnóstico precoce e prevenção de complicações associadas. . $^{1,47}$

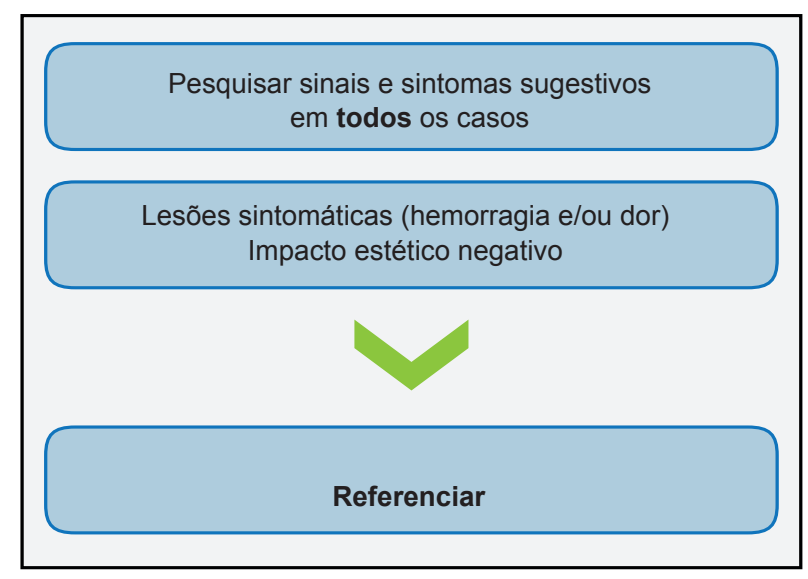

Figura 4 - Fluxograma de investigação e referenciação à consulta de Dermatologia

\section{CONCLUSÃO}

Sendo definida como uma doença rara, a abordagem sistematizada e uniformizada do doente torna-se crucial no rastreio, diagnóstico e prevenção das complicações associadas à $\mathrm{THH}$, assim como na melhor compreensão da doença. Como tal, através das propostas de referenciação hospitalar apresentadas no presente artigo, os autores

Ponderar estudo genético em todos os doentes com a suspeita ou diagnóstico de $\mathrm{THH}$

1. Doentes com diagnóstico clínico de THH, para identificar mutação familiar;

2. Doentes com suspeita de THH que:

- não cumprem os critérios clínicos suficientes para diagnóstico;

- são assintomáticos ou paucissintomático mas com familiares de $1^{\circ}$ grau com mutação conhecida, principalmente para:

a) Rastreio pré-natal em possíveis portadores;

b) Crianças com progenitores afectados.

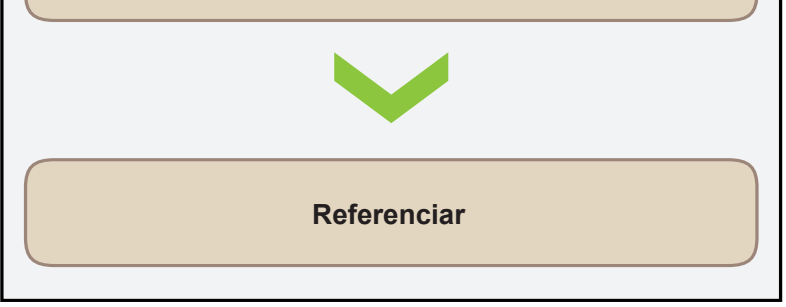

Figura 5 - Fluxograma de investigação e referenciação à consulta de Genética Médica 
oferecem um contributo importante na prática clínica de profissionais de várias especialidades hospitalares.

\section{CONTRIBUTO DOS AUTORES}

IC: Aquisição, análise e interpretação de dados. Redação e revisão crítica do artigo.

IG, SM, MJA, BR, RC: Contribuição substancial para a concepção do trabalho. Redação do artigo e aprovação da versão final.

LD: Revisão crítica do trabalho e aprovação da versão final.

\section{REFERÊNCIAS}

1. Govani F, Shovlin C. Hereditary haemorrhagic telangiectasia: a clinical and scientific review. Eur J Hum Genet. 2009;17:860-71.

2. Zarrabeitia R, Fariñas-Álvarez C, Santibáñez M, Señaris B, Fontalba A, Botella LM, el al. Quality of life in patients with hereditary haemorrhagic telangiectasia (HHT). Health Qual Life Outcomes. 2017;15:19.

3. Shovlin C, Guttmacher A, Buscarini E, Faughnan M, Hyland R, Westermann C, et al. Diagnostic criteria for hereditary hemorrhagic telangiectasia (Rendu-Osler-Weber syndrome). Am J Med Genet. 2000;91:66-7.

4. Faughnan M, Palda V, Garcia-Tsao G, Geisthoff U, McDonald J, Proctor $D$, et al. International guidelines for the diagnosis and management of hereditary haemorrhagic telangiectasia. J Med Genet. 2011;48:73-87.

5. Hoag JB, Terry P, Mitchell S, Reh D, Merlo CA. An epistaxis severity score for hereditary hemorrhagic telangiectasia. Laryngoscope. 2010;120:838-43.

6. Hoag J, Terry P, Mitchell S, Reh D, Merlo C. An epistaxis severity score for hereditary hemorrhagic telangiectasia. Laryngoscope. 2010;120:83843.

7. Faughnan M, Mager J, Hetts S, Palda V, Lang-Robertson K, Buscarini $\mathrm{E}$, et al. Second International Guidelines for the Diagnosis and

\section{AGRADECIMENTOS}

A Valerie Lund, Professora Emérita de Rinologia na University College London e médica Otorrinolaringologista no Royal National Throat Nose and Ear Hospital pelo apoio na concepção do presente protocolo através da sua vasta experiência clínica na área.

\section{CONFLITOS DE INTERESSE}

Os autores declaram não ter conflitos de interesses relacionados com o presente trabalho.

Management of Hereditary Hemorrhagic Telangiectasia. Ann Intern Med. 2020;173:989-1001.

8. Curie A, Lesca G, Cottin V, Edery P, Bellon G, Faughnan M, et al. Long-term follow-up in 12 children with pulmonary arteriovenous malformations: confirmation of hereditary hemorrhagic telangiectasia in all cases. J Pediatr. 2007;151:299-306.

9. Easey A, Wallace G, Hughes J, Jackson J, Taylor W, Shovlin C. Should asymptomatic patients with hereditary haemorrhagic telangiectasia (HHT) be screened for cerebral vascular malformations? Data from 22061 years of HHT patient life J Neurol Neurosurg Psychiatry. 2003;74:743-8

10. Eker O, Boccardi E, Sure U. European Reference Network for Rare Vascular Diseases (VASCERN) position statement on cerebral screening in adults and children with hereditary haemorrhagic telangiectasia (HHT). Orphanet J Rare Dis. 2020;15:165.

11. Stokes $P$, Rimmer J. Intranasal bevacizumab in the treatment of HHT -related epistaxis: a systematic review. Rhinology. 2018;56:3-10.

12. Barbosa A, Hans-Filho G, Vicari C, Medeiros M, Couto D, Takita L. Rendu-Osler-Weber syndrome: dermatological approach. An Bras Dermatol. 2015;90:S226-8 